\title{
Factors Associated with Increased Caregiver Burden of Informal Caregivers during the COVID-19 Pandemic in Japan
}

\author{
Y. Otobe ${ }^{l}$, Y. Kimura ${ }^{1,2}$, M. Suzuki ${ }^{1}$, S. Koyama ${ }^{1}$, I. Kojimal ${ }^{\text {, M. Yamada }}{ }^{3}$

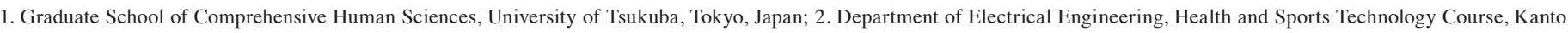 \\ Gakuin University, Japa; 3. Faculty of Human Sciences, University of Tsukuba, Tokyo, Japan
}

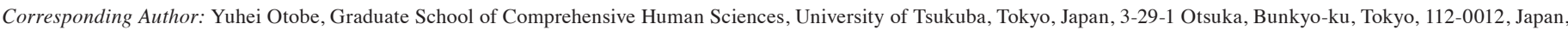
Tel: +81-29-853-2111, E-mail: otobeyuhei@gmail.com

\begin{abstract}
This study's objective was to explore the association between various factors and the increased caregiver burden of informal caregivers during the COVID-19 pandemic. On February, 2021, 700 informal caregivers completed an online survey. We assessed the change in caregiver burden during the COVID-19 pandemic. Among all caregiver participants, $287(41.0 \%)$ complained of an increased caregiver burden due to the COVID-19 pandemic. The factors associated with increased caregiver burden were depressive symptoms in caregivers [odds ratio (OR), 2.20; 95\% confidence interval (CI), 1.50-3.23], dementia (OR, 2.48; 95\%CI, 1.07-5.73) and low Barthel Index scores $(\mathrm{OR}, 2.01 ; 95 \% \mathrm{CI}, 1.39-2.90)$ in care receivers, care days (OR, 1.09; 95\%CI, 1.01-1.17) and times (OR, 1.06; 95\%CI, 1.01$1.10)$, and use of home care service (OR, 1.46; 95\%CI, 1.01-2.10) and visiting care service (OR, 1.71; 95\%CI, 1.20-2.45). These findings suggest we need to pay attention to the physical and mental health of both the care receivers and caregivers.
\end{abstract}

Key words: COVID-19, caregiver burden, informal caregivers, factors.

\section{Introduction}

The coronavirus disease 2019 (COVID-2019) pandemic has restricted a variety of social activities and has also seriously affected geriatric care and welfare. In this context, the growing caregiver burden of informal (family) caregivers has become increasingly serious. Several studies have reported that the caregiver burden of informal caregivers increased as a result of the COVID-19 pandemic (1-3). The causes of increased caregiver burden during the COVID-19 may not only be related to a decline in cognitive function, physical function, and activities of daily living (ADL) in older care receivers (2-4), but in addition it has become difficult to make use of home care and/or visiting care services because of the pandemic. However, the main cause of the increase in caregiver burden is still unclear, even after considering a variety of factors, including those related to caregivers, care receivers, and care information. The aim of this study was therefore to explore the association between various factors and the increased caregiver burden of informal caregivers during the COVID-19 pandemic in Japan.

\section{Methods}

\section{Participants}

From February 4 to 7, 2021 during the third wave of COVID-19 in Japan, an online survey was completed by 700 informal caregivers who were 20 to 89 years old and had a familial relationship with a care receiver who was a spouse, parent or parent in-law. The participants of this study were recruited through convenient sampling by online research agency. This study was conducted in accordance with the guidelines proposed by the Declaration of Helsinki, and the study protocol was reviewed and approved by the Ethics Committee of the Faculty of Human Sciences, University of Tsukuba. Electronic informed consent was obtained from all participants.

\section{Change in caregiver burden}

We assessed changes in caregiver burden during the COVID19 pandemic. The caregivers were asked, "Has your caregiver burden increased as a result of the COVID-19 pandemic?", to which they answered with "Yes" or "No."

\section{Characteristics}

We assessed the demographic characteristics of both caregivers and care receivers. For caregivers, the following items were assessed: age, sex, body mass index (BMI), presence of depressive symptoms, and frailty. Depressive symptoms were measured using the 15-item Geriatric Depression Scale (GDS-15) (5) in caregivers aged over 65 years, and using the Self-Rating Depression Scale (SDS) (6) in caregivers aged less than 65 years. We defined caregivers with GDS $\geq 5$ or with SDS $\geq 40$ as showing depressive symptoms $(7,8)$. Frailty status among caregivers was assessed using the Kihon checklist (9), and we defined scores of 8 or more as frailty, according to a previous study (10). Similarly, we assessed data across several characteristics for care receivers, including age, sex, BMI, relationship to caregiver (spouse, parent, parent-in-law), comorbidities (cancer, heart failure, stroke, diabetes, falls and fractures, neurological disorder, 


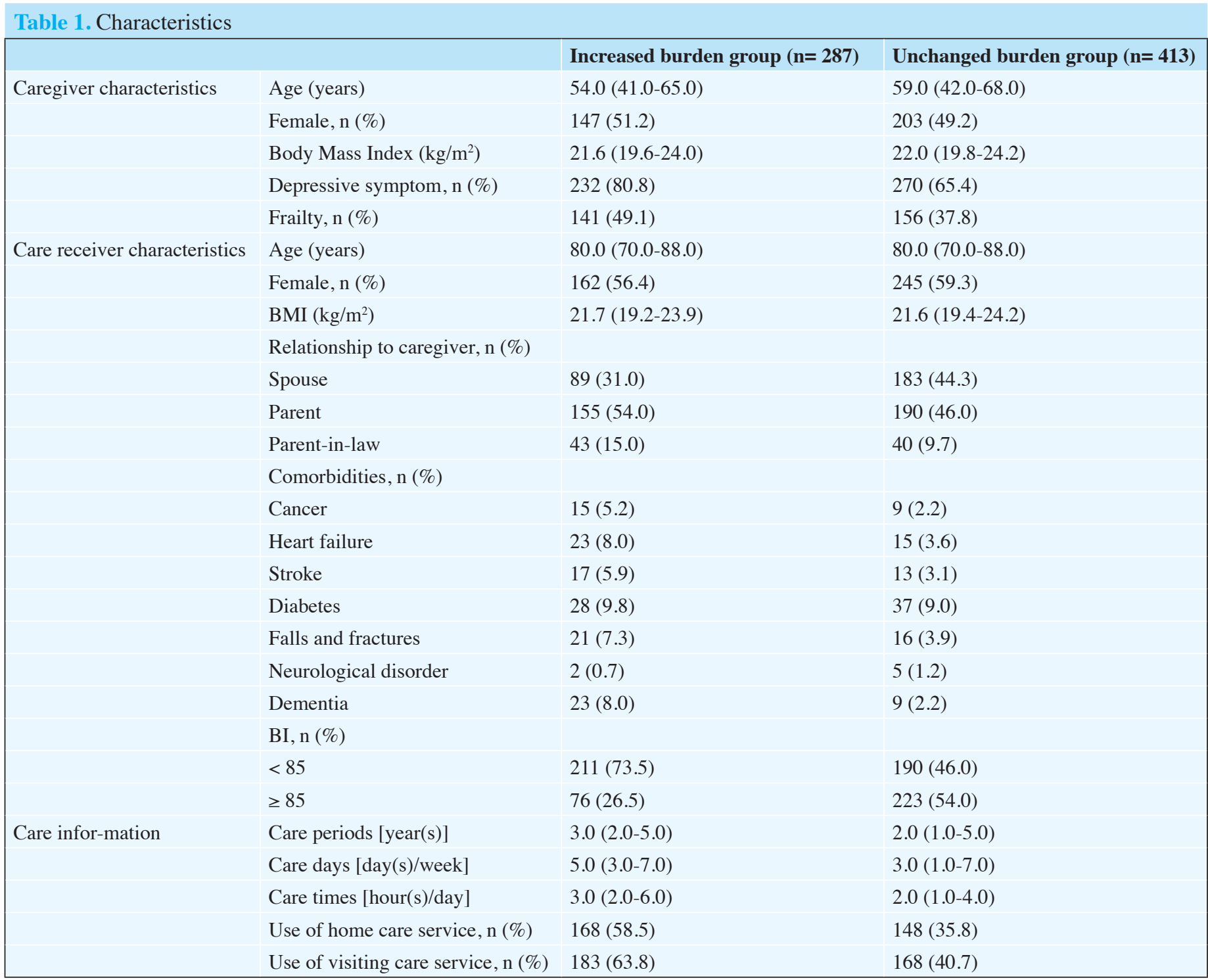

All indicators are expressed in median (IQR) aside from the categorical data; BMI, body mass index; BI, Barthel index

and dementia), and Barthel index (BI) score. The higher BI scores represent better ADL. Additionally, we investigated care information such as care period [year(s)], care days [day(s) per week], care times [hour(s) per day], use of home and visiting care services (including nursing, rehabilitation, and day service). All questions were answered by caregivers.

\section{Statistical analysis}

We examined the factors associated with the change in caregiver burden during the COVID-19 pandemic using univariate and multivariate logistic regression analyses with a stepwise forward selection method. The data were analyzed using IBM SPSS Statistics for Mac (version 27.0; IBM Japan, Tokyo, Japan). A P-value of less than 0.05 was considered to indicate statistical significance for all analyses.

\section{Results}

The characteristics are presented in Table 1. Among the total caregivers, $287(41.0 \%)$ and $413(59.0 \%)$ were in the increased and unchanged caregiver burden due to COVID-19 pandemic groups, respectively. The results of the univariate logistic regression analysis and stepwise forward selection to determine the factors associated with increased caregiver burden due to the COVID-19 pandemic are summarized in Table 2. All indicators in this study were entered into the stepwise forward selection and several factors were chosen, as follows: caregivers with depressive symptoms (odds ratio [OR]: $2.20,95 \%$ confidence interval $[\mathrm{CI}]: 1.50-3.23)$, care receivers with dementia (OR: 2.48, 95\%CI: 1.07-5.73), care receiver's with BI scores of less than 85 (OR: 2.01, 95\%CI: 1.39-2.90), care days (OR:1.09, 95\%CI:1.01-1.17), care times (OR:1.46, 95\%CI: 1.01-2.10), use of home care service (OR:1.46, 95\%CI: 1.01-2.10), and use of visiting care service (OR:1.71, 95\%CI: 1.20-2.45). 
Table 2. Univariate and multiple logistic regression analysis with stepwise forward selection to determine the factors associated with increased caregiver burden during the COVID-19 pandemic

\begin{tabular}{|c|c|c|c|c|c|c|}
\hline & \multirow[b]{2}{*}{ Variables } & \multirow[b]{2}{*}{ Units of Increase } & \multicolumn{2}{|c|}{ Univariate } & \multicolumn{2}{|c|}{ Stepwise forward selection } \\
\hline & & & OR & $95 \% \mathrm{CI}$ & OR & $95 \% \mathrm{CI}$ \\
\hline \multirow[t]{5}{*}{ Caregiver char-acteristics } & Age & 1year & 0.99 & $0.98-0.99$ & & \\
\hline & Sex (ref: male) & - & 1.09 & $0.80-1.47$ & & \\
\hline & BMI & $1 \mathrm{~kg} / \mathrm{m}^{2}$ & 0.97 & $0.93-1.02$ & & \\
\hline & Depressive symptom & - & 2.23 & $1.56-3.19$ & 2.20 & $1.50-3.23$ \\
\hline & Frailty & - & 1.59 & $1.17-2.16$ & & \\
\hline \multirow[t]{15}{*}{ Care receiver characteristics } & Age & 1year & 1.00 & $0.99-1.01$ & & \\
\hline & Sex (ref: male) & - & 0.89 & $0.66-1.21$ & & \\
\hline & BMI & $1 \mathrm{~kg} / \mathrm{m}^{2}$ & 1.01 & $0.97-1.05$ & & \\
\hline & Relationships (ref: spouse) & & & & & \\
\hline & Parent & - & 1.68 & $1.21-2.33$ & & \\
\hline & Parent-in-law & - & 2.21 & $1.34-3.64$ & & \\
\hline & Comorbidities & & & & & \\
\hline & Cancer & - & 2.48 & $1.07-5.74$ & & \\
\hline & Heart failure & - & 2.31 & $1.18-4.51$ & & \\
\hline & Stroke & - & 1.93 & $0.93-4.05$ & & \\
\hline & Diabetes & - & 1.10 & $0.66-1.84$ & & \\
\hline & Falls and fractures & - & 1.96 & $1.00-3.82$ & & \\
\hline & Neurological disorder & - & 0.57 & $0.11-2.97$ & & \\
\hline & Dementia & - & 3.91 & $1.78-8.58$ & 2.48 & $1.07-5.73$ \\
\hline & BI (ref: $\geq 85$ ) & - & 3.26 & $2.35-4.51$ & 2.01 & $1.39-2.90$ \\
\hline \multirow[t]{5}{*}{ Care infor-mation } & Care periods & 1year & 1.00 & $0.98-1.02$ & & \\
\hline & Care days & 1day & 1.18 & $1.11-1.26$ & 1.09 & $1.01-1.17$ \\
\hline & Care times & 1hour & 1.12 & $1.08-1.17$ & 1.06 & $1.01-1.10$ \\
\hline & Use of home care service & - & 2.53 & $1.86-3.44$ & 1.46 & $1.01-2.10$ \\
\hline & Use of visiting care service & _ & 2.57 & $1.88-3.50$ & 1.71 & $1.20-2.45$ \\
\hline
\end{tabular}

OR, odds ratio; CI, confidence interval; BMI, body mass index; BI, Barthel index

\section{Discussion}

In this study, $41 \%$ of caregivers complained of an increase in caregiver burden due to the COVID-19 pandemic. We found that factors associated with increased caregiver burden during the COVID-19 pandemic were caregivers with depressive symptoms, care receivers with dementia and low BI scores, care days and times, and use of home care and visiting care services.

These results might be caused by increased caregiving intensity in the pandemic situation; however, the risk of increasing caregiver burden has been present from before the onset of the pandemic. Previous studies conducted before the pandemic reported that caregivers with depressive symptoms and who cared for individuals with dementia and low ADL were more likely to experience increased caregiver burden than caregivers who did not experience these factors (1114). Therefore, caregivers whose caregiver burden increased during the COVID-19 in this study may have had pre-existing risk factors for high caregiver burden before the pandemic. Additionally, Cohen et al. reported that caregiving intensity increased significantly as a result of the COVID-19 pandemic
(15). Caregivers who had increased caregiving intensity were more likely to complain of feelings of depression and anxiety than those who did not have increased caregiving intensity (16). This would be caused by not only the health condition of care receivers, such as worsening of cognitive symptoms (17), but also by changes in the care situation with little external support. Hence, various factors before and during the pandemic may have had complex interactions and influenced the increased caregiver burden.

Moreover, care information such as care days, care times, and use of home care and visiting care services were associated with increased caregiver burden during the COVID-19 pandemic, as well as caregiver and care receiver characteristics. An increase in care days and care times, together with the use of home care and visiting care services, may mean higher care dependency among care receivers. During the pandemic, many informal caregivers had difficulty obtaining adequate care from outside the household because of reduced availability of paid services and care support, and/or they requested a break in such services because of COVID-19-related infection control measures $(16,18)$. Therefore, informal caregivers had to had to compensate for insufficient provision of home-based care 
by themselves, which might have contributed to their increased caregiver burden during the COVID-19 pandemic.

The present study has some limitations. First, this study is cross-sectional in nature and, therefore, the longitudinal causal relationship between the COVID-19 pandemic and increased caregiver burden remains unclear. Second, this study was conducted only in Japan; however, the situation of the COVID19 differs by country and region. Therefore, further research is needed to generalize our findings in other countries.

In conclusion, many informal caregivers complained of an increase in caregiver burden due to the COVID-19 pandemic. In addition, caregivers with depressive symptoms, care receivers with dementia and low BI scores, care days and times, and use of home care and visiting care services were associated with an increase in caregiver burden. These results suggest that we need to pay attention to the physical and mental health of not only care receivers but also caregivers under the "new normal" conditions of the COVID-19 pandemic.

Disclosures: The authors have no potential conflicts of interest to disclose.

Conflict of interest: None

Ethical Standards: The ethics committee approved the study.

\section{References}

1. Cohen G, Russo MJ, Campos JA, Allegri RF. Living with dementia: increased leve of caregiver stress in times of COVID-19. Int Psychogeriatr. 2020;32(11):1377-1381. doi:10.1017/S1041610220001593

2. Tsapanou A, Papatriantafyllou JD, Yiannopoulou K, et al. The impact of COVID-19 pandemic on people with mild cognitive impairment/dementia and on their caregivers. Int J Geriatr Psychiatry. 2021;36(4):583-587. doi:10.1002/gps.5457

3. Borges-Machado F, Barros D, Ribeiro Ó, Carvalho J. The Effects of COVID-19 Home Confinement in Dementia Care: Physical and Cognitive Decline, Severe Neuropsychiatric Symptoms and Increased Caregiving Burden. Am J Alzheimers Dis Other Demen. 2020;35:1533317520976720
4. Kazawa K, Kubo T, Ohge H, Akishita M, Ishii S. Preparedness guide for people with dementia and caregivers in COVID-19 pandemic. Geriatr Gerontol Int 2021;21(7):593-595. doi:10.1111/ggi.14178

5. Yesavage JA, Sheikh JI: Geriatric Depression Scale (GDS). Recent evidence and development of a shorter version. Clin Geron 5:165-173, 1986

6. Zung WW. A SELF-RATING DEPRESSION SCALE. Arch Gen Psychiatry. 1965 Jan;12:63-70.

7. Zung WW. How normal is depression? Psychosomatics. 1972 May-Jun;13(3):174-8.

8. Lyness JM, Noel TK, Cox C, King DA, Conwell Y, Caine ED. Screening for depression in elderly primary care patients. A comparison of the Center for Epidemiologic Studies-Depression Scale and the Geriatric Depression Scale. Arch Intern Med. 1997 Feb 24;157(4):449-54.

9. Arai H, Satake S. English translation of the Kihon Checklist. Geriatr Gerontol Int 2015; 15: 518-9.

10. Satake S, Senda K, Hong YJ, et al. Validity of the Kihon Checklist for assessing frailty status. Geriatr Gerontol Int. 2016 Jun;16(6):709-15

11. Grov EK, Fosså SD, Sørebø O, Dahl AA. Primary caregivers of cancer patients in the palliative phase: a path analysis of variables influencing their burden. Soc Sci Med. 2006;63(9):2429-2439.

12. Beach SR, Schulz R, Williamson GM, Miller LS, Weiner MF, Lance CE. Risk factors for potentially harmful informal caregiver behavior. J Am Geriatr Soc. 2005;53(2):255-261.

13. Connors MH, Seeher K, Teixeira-Pinto A, Woodward M, Ames D, Brodaty H. Dementia and caregiver burden: A three-year longitudinal study. Int J Geriat Psychiatry. 2020;35(2):250-258. doi:10.1002/gps.5244

14. Gallicchio L, Siddiqi N, Langenberg P, Baumgarten M. Gender differences in burden and depression among informal caregivers of demented elders in the community. Int $\mathrm{J}$ Geriatr Psychiatry. 2002;17(2):154-163.

15. Cohen SA, Kunicki ZJ, Drohan MM, Greaney ML. Exploring Changes in Caregiver Burden and Caregiving Intensity due to COVID-19. Gerontol Geriatr Med. 2021;7:2333721421999279.

16. Bergmann M, Wagner M. The Impact of COVID-19 on Informal Caregiving and Care Receiving Across Europe During the First Phase of the Pandemic. Front Public Health. 2021;9:673874. Published 2021 Jun 16. doi:10.3389/fpubh.2021.673874

17. Canevelli M, Valletta M, Toccaceli Blasi M, et al. Facing Dementia During the COVID-19 Outbreak. J Am Geriatr Soc. 2020;68(8):1673-1676. doi:10.1111/jgs.16644

18. Todorovic N, Vracevic M, Rajovic N, et al. Quality of Life of Informal Caregiver behind the Scene of the COVID-19 Epidemic in Serbia. Medicina (Kaunas). 2020;56(12):647. Published 2020 Nov 26. doi:10.3390/medicina56120647

How to cite this article: Y. Otobe, Y. Kimura, M. Suzuki, et al. Factors Associated with Increased Caregiver Burden of Informal Caregivers during the COVID-19 Pandemic in Japan. J Nutr Health Aging. 2022;26(2):157-160, http://dx.doi.org/10.1007/s12603022-1730-y 